УДК 519.41/47

\title{
Some Minimal Conditions in Certain Extremely Large Classes of Groups
}

\author{
Nikolai S. Chernikov* \\ Institute of Mathematics \\ National Academy of Sciences of Ukraine \\ Tereschenkivska, 3, Kyiv-4, 01601
}

Ukraine

Received 10.10.2014, received in revised form 10.11.2014, accepted 26.12.2014

$\overline{\text { Let } \mathfrak{L} \text { (respectively } \mathfrak{T}) \text { be the minimal local in the sense of D. Robinson class of groups, containing the }}$ class of weakly graded (respectively primitive graded) groups and closed with respect to forming subgroups and series. In the present paper, we completely describe: the $\mathfrak{L}$-groups with the minimal conditions for non-abelian subgroups and for non-abelian non-normal subgroups; the $\mathfrak{T}$-groups with the minimal conditions for (all) subgroups and for non-normal subgroups. By the way, we establish that every $\overline{I H}$ group, belonging to $\mathfrak{L}$, is solvable.

Keywords: local classes of groups; minimal conditions; non-abelian, Chernikov, Artinian, Dedekind, $\overline{I H}$-groups; weakly, locally, binary, primitive graded groups.

\section{Introduction. Some preliminary data}

In the present paper, the author continues his investigations [1-5]. Remind that the class $\mathfrak{X}$ of groups is called local (in our sense), if it includes every group that has a local system of subgroups belonging to $\mathfrak{X}$ or, in the other words, a local system of $\mathfrak{X}$-subgroups (see [1]). Further, introduce the definition.

Definition. The class $\mathfrak{X}$ of groups will be called local in the sense of D. Robinson, if it includes every group $G$ such that for any finite set $F$ of elements of $G$, there exists some $\mathfrak{X}$-subgroup $S$ of $G$, containing $F$.

(In connection with this definition, see [6, p. 93].)

The following useful elementary lemmas hold.

Lemma 1. Assume that some local class $\mathfrak{X}$ of groups is closed with respect to forming subgroups. Then $\mathfrak{X}$ is local in the sense of D. Robinson.

Proof. Let $G, F$ and $S$ be from Definition. Since $\langle F\rangle \subseteq S \in \mathfrak{X},\langle F\rangle \in \mathfrak{X}$. Thus all finitely generated subgroups of $G$ form its local system of $\mathfrak{X}$-subgroups. Consequently, $G \in \mathfrak{X}$.

Remind that the class $\mathfrak{X}$ of groups is closed with respect to forming series, if every group, having a series with $\mathfrak{X}$-factors, belongs to $\mathfrak{X}$. Sometimes a series is also called a generalized normal system (S. N. Chernikov).

Lemma 2. Let $\mathfrak{X}$ and $\mathfrak{Y}$ be respectively the minimal local and the minimal local in the sense of D. Robinson classes of groups, containing some fixed class $\mathfrak{V}$ of groups and closed with respect to forming subgroups and series (generalized normal systems). Then $\mathfrak{X}=\mathfrak{Y}$.

*chern@imath.kiev.ua

(c) Siberian Federal University. All rights reserved 
Proof. Indeed, $\mathfrak{X}$ (respectively $\mathfrak{Y}$ ) is the intersection of all local (respectively all local in the sense of D.Robinson) classes of groups, containing $\mathfrak{V}$ and closed with respect to forming subgroups and series. Since all local in the sense of D. Robinson classes of group are local, obviously, $\mathfrak{X} \subseteq \mathfrak{Y}$. But in view of Lemma $1, \mathfrak{X}$ is local in the sense of $\mathrm{D}$. Robinson. Thus $\mathfrak{X}=\mathfrak{Y}$. Lemma is proven.

Remind that the group $G$ is called weakly graded, if for $g, h \in G$, the subgroup $<g, g^{h}>$ possesses a subgroup of finite index $\neq 1$ whenever $|\langle g\rangle|=\infty$, or $g$ is a $p$-element $\neq 1$ with some odd prime $p$ and also $\left[g^{p}, h\right]=1$ and the subgroup $\langle g, h\rangle$ is periodic (N. S. Chernikov $[2$, P. 22]). The class of weakly graded groups is very large and includes, for instance,: the classes of binary graded, locally graded, binary finite, locally finite, locally solvable groups; the classes of linear groups, 2-groups, periodic Shunkov groups; all Kurosh-Chernikov classes of groups.

Remind that the group $G$ is called primitive graded, if for $g, h \in G$, the subgroups $<g, g^{h}>$ possesses a subgroup of finite index $\neq 1$ whenever $g$ is a $p$-element $\neq 1$ with some odd $p$ and also $\left[g^{p}, h\right]=1$ and $\langle g, h\rangle$ is periodic (N.S. Chernikov [1]). The class of weakly graded groups is obviously a subclass of the class of primitive graded groups. Further, every Ol'shanskiy's infinite simple torsion-free group with cyclic proper subgroups (see, for instance, [7] or [8, Theorem 28.3]) is primitive graded but is not weakly graded. Thus, it is a proper subclass.

Also remind: an infinite non-abelian group with normal infinite non-abelian subgroups is called an $\overline{I H}$-group (S. N. Chernikov, see, for instance, [9]). S.N.Chernikov has obtained a lot of principal results on $\overline{I H}$-groups (see, for instance, [9, Chapter 6$]$ ).

Remind that by definition the group satisfies the minimal condition for some subgroups, if it has not a descending infinite chain of these subgroups. Below $\min -\overline{a b}$ and $\min -\overline{a b} \bar{n}$ are the minimal conditions for non-abelian and non-abelian non-normal subgroups respectively; see, for instance, [2, p. 23]. (Clearly, abelian groups satisfy min $-\overline{a b}$ ). Also $\min -\bar{n}$ is the minimal condition for non-normal subgroups (see, for instance, [1], [2, p. 23]). The groups, satisfying the minimal condition for (all) subgroups, are called Artinian.

Remind that a group, in which all subgroups are normal, is called Dedekind. The Dedekind groups are exactly the abelian groups and the groups $G=Q \times T \times R$ with a quaternion group $Q$, an elementary abelian 2-group $T$ and an abelian group $R$ with all elements of odd orders (R. Baer's Theorem, see [10]).

\section{The main results}

The following very general theorems are the main results of the present paper.

Theorem 1. Let $\mathfrak{L}$ be the minimal local in the sense of D.Robinson class of groups, containing the class $\mathfrak{L}_{\mathrm{o}}$ of weakly graded groups and closed with respect to forming subgroups, series (and, at the same time, to forming subcartesian products). The non-abelian group $G \in \mathfrak{L}$ satisfies min- $\overline{a b}$ (respectively min - $\overline{a b} \bar{n}$ ) iff it is Chernikov (respectively a Chernikov group or a solvable group with normal non-abelian subgroups).

Theorem 2. Let $\mathfrak{T}$ be the minimal local in the sense of D.Robinson class of groups, containing the class $\mathfrak{L}_{0}$ of primitive graded groups and closed with respect to forming subgroups, series (and, at the same time, to forming subcartesian products). The group $G \in \mathfrak{T}$ is Artinian (respectively satisfies min $-\bar{n}$ ) iff it is Chernikov (respectively Chernikov or Dedekind).

Theorem 3. Let $\mathfrak{L}$ be the same as in Theorem $1, G$ be an $\overline{I H}$-group and $R$ be the intersection of all infinite non-abelian subgroups of $G$. The group $G$ is solvable iff $R \in \mathfrak{L}$. 
The Ol'shanskiy's Examples of infinite simple groups $G$, in which every proper non-identity subgroup has a prime order (see, for instance, [11]) show: in Theorems 1-3, the conditions:

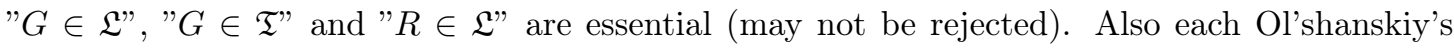
infinite simple torsion-free group with cyclic proper subgroups (see, for instance [7] or [8, Theorem 28.3]) is primitive graded, satisfies $\min -\overline{a b}, \min -\overline{a b} \bar{n}$ and is not Chernikov or solvable. Thus,

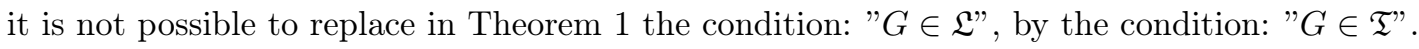

\section{Proofs of the main results}

Proof of Theorem 1. First, by virtue of Lemma 2, $\mathfrak{L}$ is the minimal local (in our) sense class of groups, containing the class $\mathfrak{L}_{0}$ and closed with respect to forming subgroups and series. Then in consequence of Lemma $1.37[6], \mathfrak{L}$ is closed with respect to forming subcartesian products. For ordinals $\alpha \neq 0$ define by induction: if for some ordinal $\beta, \alpha=\beta+1$, then $\mathfrak{L}_{\alpha}$ is the class of all groups with a local system of subgroups, possessing a series with $\mathfrak{L}_{\beta}$-factors; otherwise $\mathfrak{L}_{\alpha}=\bigcup_{\beta<\alpha} \mathfrak{L}_{\beta}$. The class $\mathfrak{L}_{0}$ is, of course, closed with respect to forming subgroups. Assume that for some $\alpha>0$, all $\mathfrak{L}_{\beta}, \beta<\alpha$, are closed with respect to forming subgroups. Then it is easy to see: $\mathfrak{L}_{\alpha}$ is also closed with respect to forming subgroups. Thus, all classes $\mathfrak{L}_{\alpha}$ and, at the same time, their union $\mathfrak{Q}=\bigcup_{\alpha} \mathfrak{L}_{\alpha}$ are closed with respect to forming subgroups. Let a group $F$ have some series with $\mathfrak{Q}$-factors. For each factor of the series, take some ordinal $\alpha$ such that $\mathfrak{L}_{\alpha}$ contains it. For the set (of cardinality $\leqslant|F|$ ) of all taken ordinals, there exists some ordinal $\zeta$ such that $\alpha<\zeta$ whenever $\alpha$ belongs to this set. Then all factors of the series belong to $\mathfrak{L}_{\zeta}$. Therefore $F \in \mathfrak{L}_{\zeta+1}$, (i.e. $F \in \mathfrak{Q}$ ). Now let a group $F$ have a local system of subgroups $K \in \mathfrak{Q}$. For each $K$, take some ordinal $\alpha$ such that $\mathfrak{L}_{\alpha}$ contains $K$. For the set of all taken ordinals, there exists some ordinal $\zeta$ such that $\alpha<\zeta$ whenever $\alpha$ belongs to this set. Then all $K$ belong to $\mathfrak{L}_{\zeta}$. Therefore $F \in \mathfrak{L}_{\zeta+1}$, (i.e. $F \in \mathfrak{Q}$ ).

Since $\mathfrak{L}_{\mathcal{o}} \subseteq \mathfrak{Q} \subseteq \mathfrak{L}$ and $\mathfrak{Q}$ is closed with respect to forming subgroups, series, and also $\mathfrak{Q}$ is local, we have: $\mathfrak{Q}=\mathfrak{L}$.

Suppose that there exist some non-abelian non-Chernikov groups $G \in \mathfrak{L}$ with $\min -\overline{a b}$. Let $\eta$ be minimal among all ordinals $\iota$, for which $\mathfrak{L}_{\iota}$ includes such $G$. In view of Theorem B [12], $\eta>0$. It is easy to see: for some ordinal $\lambda, \eta=\lambda+1$. Therefore such $G$ from $\mathfrak{L}_{\eta}$ possesses a local system of subgroups $S$ having a series with $\mathfrak{L}_{\lambda}$-factors. Every factor, clearly, satisfies $\min -\overline{a b}$ and so is Chernikov or abelian. Obviously, a series has a refinement with finite factors. Then by virtue of S. N. Chernikov's is Theorem 6.1 [9], $S$ is Chernikov or abelian. Since $G$ is not abelian, all its non-abelian subgroups $S$ form a local system. Consequently, $G$ is locally finite. Then in view of Shunkov's Theorem [13], $G$ must be Chernikov, which is a contradiction.

Suppose that there exist non-abelian non-Chernikov groups $G \in \mathfrak{L}$ with $\min -\overline{a b} \bar{n}$, which are not solvable with normal non-abelian subgroups. Let $\eta$ be as above. In view of Theorem 1 [2], $\eta>0$. It easy to see: for some ordinal $\lambda, \eta=\lambda+1$. Therefore such $G$ from $\mathfrak{L}_{\eta}$ possesses a local system of subgroups $S$ having a series with $\mathfrak{L}_{\lambda}$-factors. Every factor, clearly, satisfies min $-\overline{a b} \bar{n}$ and so is Chernikov or solvable. Therefore, obviously, a series has a refinement with finite factors. Further, any finitely generated non-identity subgroup $M$ of $G$ belongs to some $S$. Consequently $M$ has a series with finite factors. Therefore obviously $M$ has a subgroup of finite index $\neq 1$. Thus, $G$ is locally graded. Then in view of Corollary 8 [2], $G$ is a Chernikov group or a solvable group with normal non-abelian subgroups, which is a contradiction. Theorem is proven.

Proof of Theorem 2. First, by virtue of Lemma 2, $\mathfrak{T}$ is the minimal local (in our sense) 
class of groups, containing $\mathfrak{L}_{0}$ and closed with respect to forming subgroups and series. Then in consequence of Lemma 1.37 [??], $\mathfrak{T}$ is closed with respect to forming subcartesian products. The class $\mathfrak{L}_{0}$ is, of course, closed with respect to forming subgroups. Let $\mathfrak{L}_{\alpha}, \alpha>0$, and $\mathfrak{Q}$ be the same as in the proof of Theorem 1. Then: $\mathfrak{L}_{\alpha}$ and $\mathfrak{Q}$ are closed with respect to forming subgroups, $\mathfrak{Q}$ is closed with respect to forming series and $\mathfrak{Q}$ is local, and $\mathfrak{Q}=\mathfrak{T}$ (see the proof of Theorem 1).

Suppose that there exist Artinian non-Chernikov groups $G \in \mathfrak{T}$. Let $\eta$ be minimal among all ordinal $\iota$, for which $\mathfrak{L}_{\iota}$ includes such $G$. Every Artinian primitive graded group is periodic and, at the same time, weakly graded. In view of Theorem B [12], Artinian weakly graded groups are Chernikov. Thus, $\eta>0$. It is easy to see: for some ordinal $\lambda, \eta=\lambda+1$. Therefore the Artinian non-Chernikov group $G$ from $\mathfrak{L}_{\eta}$ possesses a local system of some subgroup $S$ having a series with $\mathfrak{L}_{\lambda}$-factors. Since $S$ is Artinian, the series is ascending. Every its factor is Artinian and so Chernikov. Then in consequence of O. Yu. Shmidt's Theorem (see, for instance, [6, Theorem $1.45]$ ), $S$ is locally finite. Consequently, $G$ is locally finite. Therefore in view of Shunkov-KegelWehrfritz Theorem [14,15], $G$ is Chernikov, which is a contradiction.

Let $H$ be any group with min $-\bar{n}$. If $H$ is Artinian, then $H^{\prime}$ is Artinian too. Assume that $H$ is non-Artinian. It is easy to see: $H$ contains some non-Artinian subgroup $B$ such that every non-Artinian subgroup of $B$ is normal in $H ; B$ has some descending series $B=B_{0} \supset B_{1} \supset B_{2} \supset$ $\ldots \supset B_{\gamma}=\cap_{\alpha<\gamma} B_{\alpha} \supseteq 1$ such that $B_{\gamma}$ is Artinian. Obviously all $B / B_{\alpha}, \alpha<\gamma$, are Dedekind. Consequently, in view of R. Baer's Theorem [10], $\left|\left(B / B_{\alpha}\right)^{\prime}\right| \leqslant 2, \alpha<\gamma$. Therefore $\left|\left(B / B_{\gamma}\right)^{\prime}\right| \leqslant 2$. Put $\left(B / B_{\gamma}\right)^{\prime}=D / B_{\gamma}$. Then $D$ is Artinian. Therefore $B / D$ is abelian non-Artinian. Since $H / D$ does not satisfy the minimal condition for abelian subgroups and obviously satisfies $\min -\bar{n}$, it is Dedekind (see Corollary $2[16]$ ). Put $(H / D)^{\prime}=T / D$. In view of R.Baer's Theorem [10], $|T / D| \leqslant 2$. Since $D$ is Artinian, $T$ is Artinian too. Therefore, because of $H^{\prime} \subseteq T, H^{\prime}$ is Artinian.

Now let $G$ be a $\mathfrak{T}$-group with $\min -\bar{n}$. Since $G^{\prime}$ is an Artinian $\mathfrak{T}$-group, it is Chernikov (see above). Therefore in consequence of Theorem 1 [16], $G$ is Chernikov or Dedekind. Theorem is proven.

Proof of Theorem 3. First, for every infinite non-abelian subgroup $N$ of $G$, each subgroup $H \supseteq N$ of $G$ is normal in it. Consequently, $G / N$ is Dedekind. Therefore in view of R. Baer's Theorem [10], $(G / N)^{\prime \prime}=1$. Consequently, $G^{\prime \prime} \subseteq R$.

Now assume that $R \in \mathfrak{L}$. Since each proper subgroup of $R$ is finite or abelian, $R$ satisfies min $-\overline{a b}$. Therefore in view of Theorem $1, R$ is abelian or Chernikov. In the first case, $G^{\prime \prime \prime}=1$. In the second case, $R$ contains some characteristic abelian subgroup $T$ with $|R: T|<\infty$. Clearly, $\left(C_{G}(R / T) \cap R\right) / T=Z(R / T)$. Consequently $\left(C_{G}(R / T) \cap R\right)^{\prime \prime}=1$. Since $(G / R)^{\prime \prime}=1$, we have: $\left(C_{G}(R / T) / C_{G}(R / T) \cap R\right)^{\prime \prime}=1$. Thus, $C_{G}(R / T)$ is solvable of derived length $\leqslant 4$. Further, $\left|G: C_{G}(R / T)\right|<\infty$. Let $V$ be a maximal normal solvable subgroup of $G$ containing $C_{G}(R / T)$. If $V \neq G$, then $G / V$ is non-abelian. At the same time, $G / V$ contains some non-abelian subgroup $M / V$ with abelian proper subgroups. In view of Miller-Moreno Theorem [17], $M / V$ is solvable. Then $M$ is infinite non-abelian solvable. Therefore $M$ is normal in $G$, which is a contradiction. Theorem is proven.

The following proposition was in fact established in the proofs of Theorems 1 and 2 .

Proposition. Let $\mathfrak{V}$ be some class of groups, closed with respect to forming subgroups, and $\mathfrak{K}$ be the minimal local in the sense of D. Robinson class of groups, containing $\mathfrak{V}$ and closed with respect to forming subgroups, series (and, at the same time, to forming subcartesian products). Then:

(i) if every Artinian $\mathfrak{V}$-group is Chernikov, then every Artinian $\mathfrak{K}$-group is Chernikov; 
(ii) if every $\mathfrak{V}$-group with min $-\overline{a b}$ is Chernikov or abelian, then every $\mathfrak{K}$-group with min $-\overline{a b}$ is Chernikov or abelian;

(iii) if every $\mathfrak{V}$-group with min $-\bar{n}$ is Chernikov or Dedekind, then every $\mathfrak{K}$-group with min $-\bar{n}$ is Chernikov or Dedekind;

(iv) if every $\mathfrak{V}$-group with min- $\overline{a b} \bar{n}$ is a Chernikov or abelian group, or a non-abelian solvable group with normal non-abelian subgroups, then every $\mathfrak{K}$-group with min $-\overline{a b} \bar{n}$ is the same.

\section{References}

[1] N.S.Chernikov, Primitive graded groups with the minimal condition for non-normal subgroups, Sci. J. Dragomanov Ped. Univ. Ser. 1. Phys.-math. sci. (Kiev), 2013, no. 14, 10-13.

[2] N.S.Chernikov, Groups satisfying the minimal condition for non-abelian non-normal subgroups, J. Sib. Federal Univ. Math. and Physics., 7(2014), no. 1, 22-34.

[3] N.S.Chernikov, Groups with some minimal conditions for non-normal subgroups, Proc. F. Scorina Gomel State Univ., (2014), no. 3, 121-124.

[4] N.S.Chernikov, On primitive graded groups with minimal conditions, Algebra and Applications: Trans. Intern. Conf. dedicated to the $100^{t h}$ anniversary of Prof. L.A. Kaluznin (Nalchik, Russia, September 6-11, 2014), Kabard.-Balkarian State Univ., 2014, Nalchik, $130-132$.

[5] N.S. Chernikov, Artinian groups and groups with minimal conditions for non-abelian and non-normal subgroups. Book of abstracts of the Intern. Algebraic Conf. dedicated to 100-th anniversary of L. A. Kaluznin (Kyiv, July 7-12, 2014), Taras Shevchenko Nat. Univ., 2014, Kyiv, Ukraine, 22.

[6] D.J.S.Robinson, Finiteness conditions and generalized soluble groups. Pt. 2, Springer, Berlin etc, 1972.

[7] A.Yu.Ol'shanskiy, An infinite simple Noetherian torsion-free group, Izvestiya AN SSSR. Ser. mat., 43(1979), no. 6, 1328-1393 (in Russian).

[8] A.Yu.Ol'shanskiy, Geometry of defining relations in groups, Nauka, Moskow, 1989 (in Russian).

[9] S.N. Chernikov, Groups with prescribed properties of the system of subgroups, Nauka, Moskow, 1980 (in Russian).

[10] R.Baer, Situation der Untergruppen und Struktur der Gruppe, S.-B. Heidelberg Akad. Math.-Nat. Klasse, 2(1933), 12-17.

[11] A.Yu.Ol'shanskiy, An infinite group with subgroups of prime orders, Izvestiya AN SSSR. Ser. mat., 44(1980), no. 2, 30-321 (in Russian).

[12] N.S.Chernikov, Groups with minimal conditions, J. Math. Sci., 163(2009), no. 6, 774-784. 
[13] V.P.Shunkov, On abstract characterizations of some linear groups, Algebra. Matrices and matrix groups, Institut Fiziki Sib. Otd. AKad. Nauk SSSR, Krasnoyarsk, 1970, 5-54 (in Russian).

[14] V.P.Shunkov, On the minimality problem for locally finite groups, Algebra i logika, 9(1970), no. 2, 220-248 (in Russian).

[15] O.-H.Kegel, B.A.F.Wehrfrits, Strong finiteness conditions in locally finite group, Math. Žs, 117(1970), no. 1-4, 309-324.

[16] S.N.Chernikov, The infinite non-abelian groups with the minimal condition for non-normal subgroups, Mat. Zametki, 6(1969), no. 1, 11-18 (in Russian).

[17] G.A.Miller, H.Moreno, Non-abelian groups in which every subgroup is abelian, Trans. Amer. Math. Soc., 4(1903), no. 4, 398-404.

\section{Некоторые условия минимальности в некоторых экстремально больших классах групп}

\section{Николай С. Черников}

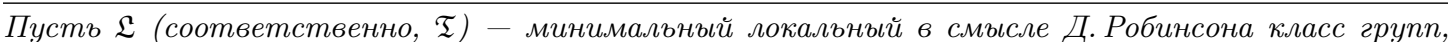
содержащий класс слабоступенчатых (соответственно, примитивно ступенчатых) групп и замкнутый относительно образования подгрупп и обобщенных нормальных систем. В настоящей работе мы полностъю описываем: $\mathfrak{L}$-группы с условиями минимальности для неабелевых подгрупп и для неабелевых ненормальных подгрупп; T-группы с условиями минимальности для (всех) подгрупп и для ненормальных подгрупп. Попутно мы устанавливаем, что любая $\overline{I H}$-группа, принадлежащая $\kappa \mathfrak{L}$, разрешима.
}

Ключевые слова: локальные классы групп; условия минимальности; неабелевъ, черниковские, артиновы, дедекиндовы, $\overline{I H}$-группъ; слабо, локалъно, бинарно, примитивно ступенчатые группь. 